\title{
Exogenèse d'un projet architectural : à la lumière des entretiens avec Fernando Montès
}

\section{Olfa Meziou Baccour}

\section{(2) OpenEdition}

1 Journals

Édition électronique

URL : https://journals.openedition.org/genesis/5721

DOI : $10.4000 /$ genesis. 5721

ISSN : 2268-1590

Éditeur :

Presses universitaires de Paris Sorbonne (PUPS), Société internationale de génétique artistique littéraire et scientifique (SIGALES)

Édition imprimée

Date de publication : 15 décembre 2020

Pagination : 149-166

ISBN : 979-10-231-0704-3

ISSN : 1167-5101

\section{Référence électronique}

Olfa Meziou Baccour, «Exogenèse d'un projet architectural : à la lumière des entretiens avec Fernando Montès », Genesis [En ligne], 51 | 2020, mis en ligne le 20 décembre 2021, consulté le 07 février 2022 URL : http://journals.openedition.org/genesis/5721 ; DOI : https://doi.org/10.4000/genesis.5721 


\title{
Exogenèse d'un projet architectural : à la lumière des entretiens avec Fernando Montès
}

\author{
Olfa Meziou Baccour
}

$\mathrm{D}$ ans le rapport intitulé Pour une analyse génétique des techniques de projet et du processus de conception en architecture - Étude de genèse des premiers dessins du projet de Fernando Montès - 20/28 rue Ramponneau Paris 1986-1990, Pierre-Marc de Biasi, l'un des deux co-auteurs (l'autre étant Fernando Montès), évoquait l'évolution possible et souhaitée de la génétique textuelle. Deux directions étaient privilégiées :

- l'élargissement des études génétiques à d'autres secteurs de la création avec pour seule condition que celle-ci ait laissé comme traces des documents déchiffrables;

- la possibilité d'utiliser des informations collectées grâce à l'observation directe et à l'entretien, informations qui souvent ne laissent pas de traces écrites ou graphiques, ce que l'auteur décrit comme «aller saisir son objet dans l'état actuel de sa genèse ».

Tout cela paraît cohérent puisque tel était l'objet même de la mission : décrire une tentative d'adaptation de la génétique textuelle à un nouveau champ de création - l'architecture - avec la collaboration de l'architecte, qui accepte de conserver et de transmettre ses documents de travail, de se laisser observer durant la conception et de donner des entretiens à ce sujet.

Le rapport consigne les résultats d'une étude portant sur les onze premiers dessins du dossier de genèse du 20/28 rue Ramponneau, et constitue le point de départ d'une étude entamée quinze ans plus tard, celle dont il est question ici. S'il n'était plus possible d' «aller saisir son objet dans l'état actuel de sa genèse », l'architecte n'a pas été considéré comme un «être de papier ${ }^{1} »$ mais comme un matériau génétique, c'est-à-dire comme une source d'indices. En effet, de nouveaux entretiens avec Fernando Montès enrichissent le dossier de genèse.

Dans notre contexte d'enquête exogénétique - si l'on peut appeler ainsi la recherche des sources et le repérage de leurs traces et de leurs transformations dans l'ante-projet, équivalent architectural de l'avant-texte -, le recours aux entretiens a permis de constater une interférence certaine avec les informations contenues dans les «brouillons» et a révélé leur caractère double, à la fois éclairant et biaisant. C'est sur ce statut de l'entretien comme élément spécifique du dossier de genèse, et sur son rôle particulier dans l'élucidation des processus exogénétique, que cette étude voudrait apporter sa contribution.

\section{Territoire de l'exogenèse. Au-delà du contexte}

Avant de commencer cette enquête exogénétique, il convient de signaler une spécificité architecturale. Elle concerne le statut de la commande, ce projet de l'autre auquel se confronte l'architecte, cette extériorité pour et en dépit de laquelle il devra inscrire son propre projet. Elle concerne aussi celui du «site», cette autre extériorité immédiate dans laquelle le projet vient prendre place. En effet, si «l'exogenèse désigne tout procès d'écriture consacré à un travail de recherche, de sélection et d'intégration qui porte sur des informations émanant d'une source extérieure à l'écriture ${ }^{2}$ », force est de constater que les éléments de la commande et les données relatives

1. «Or on est obligé de considérer que l'auteur auquel nous avons affaire dans un dossier génétique n'est lui aussi en pratique qu'un être de papier. » Daniel Ferrer, «Le matériel et le virtuel. Du paradigme indiciaire à la logique des mondes possibles », dans M. Contat et D. Ferrer (dir.), Pourquoi la critique génétique? Méthodes et théories, Paris, CNRS Éditions, 1998, p. 11-30.

2. Pierre-Marc de Biasi, «Qu'est-ce qu'un brouillon? Le cas Flaubert : essai de typologie fonctionnelle des documents de genèse », dans M. Contat et D. Ferrer (dir.), Pourquoi la critique génétique? Méthodes et théories, op. cit., p. 31-60. 
au site relèvent bel et bien de l'exogenèse. En tant que composantes textuelles et dessinées délimitant l'espace de la commande, mais aussi en tant qu'expérience vécue par l'architecte qui visite carnet en main les lieux d'implantation de son futur projet, ces données sont bien des informations émanant d'une source extérieure à la «projétation» (francisation de la projettazione italienne) que l'architecte interprète et intègre à son travail de conception ${ }^{3}$. Daniel Guibert met d'ailleurs commande, site et premières références convoquées dans une même catégorie qu'il nomme «données initiales » :

ensemble d'informations constituées par le concepteur à partir des données, contraintes et exigences du programme, dont celle du coût, des données physiques et réglementaires attachées au site : les données initiales comprennent aussi toutes les informations en provenance de la manipulation du matériel référentiel convoqué par le concepteur dès la lecture du programme, sa prise de contact avec le site et les institutions ambiantes 4 .

Toutefois, dans la mesure où, ici, «exogenèse» a été associée à «intertextualité », il convient peut-être, pour rester centré sur cette problématique, d'interroger l'exogenèse en ce qu'elle nous renseigne sur les liens à d'autres projets qui peuvent caractériser un processus de production d'une œuvre architecturale. Ces liens, malgré cette «restriction», peuvent dans certains cas être générés par la commande. Le site, surtout quand il est urbain, en crée indéniablement. Dans le projet du 20/28 rue Ramponneau, plus qu'une commande et plus qu'un site, on trouve un contexte qui a été un fort générateur d' «interprojectualité». Un petit rappel historique permet de le comprendre :

En 1974, la Ville de Paris lance une procédure de déclaration d'utilité publique (DUP) sur un grand îlot à Belleville. Le plan d'aménagement de la DUP est confié à Dominique Druenne et au Groupe Cerise. Approuvé en 1978, il propose le «retour à l'îlot» par un plan où les opérations conservent l'échelle des parcelles existantes. L'avancement du dossier est retardé et ce n'est qu'en 1986 que les premiers immeubles de Dominique Druenne, conformes au gabarit Bellevillois, sont construits. Ce seront les seuls. Entre-temps, le débat s'est développé et l'«urbanité retrouvée» n'est plus associée à la conservation de l'échelle de la parcelle. La Régie immobilière de la Ville de Paris - mise en place à l'origine pour construire des logements pour la classe moyenne - prend conscience de ce que le territoire gigantesque de Belleville est sous exploité et décide, pour l'enrichir et le moderniser, de desserrer la trame urbaine. Une seconde génération de projets est confiée à de jeunes architectes, sur des terrains enjambant plusieurs des anciennes parcelles.

Le 20-28 est confié à Fernando Montès. Celui-ci, avec ses collègues Jacques Ripault, Denise Duhart et Frédéric Borel, approuvent tout à fait les nouvelles orientations de la RIVP et voudraient développer, à l'occasion de la restructuration de cet îlot, deux notions complémentaires : le prolongement de l'espace public primaire à l'intérieur de l'immeuble et ce qu'ils appellent le continuum spatial organisé qui consisterait, en gros, à différencier le vide introduit dans l'immeuble par la création de tons d'espaces différents sur l'axe publicprivé et ce, par l'invention de dispositifs architecturaux associables à des seuils. Leur discours plaît au directeur de la RIVP, qui voit en leur collaboration une sorte de laboratoire d'intervention urbaine 5 .

Avec ce projet, nous sommes dans un cas particulier où des architectes interviennent sur un même îlot, chacun de manière individuelle, tout en réfléchissant ensemble sur les liens qu'il convient de créer. Fernando Montès est chargé de concevoir le dernier projet. Dans le document D3 daté du 6 septembre 1986, on trouve trois dessins. Sur deux d'entre eux, le projet n'est pas seul. Le premier est une vue en perspective de la façade sur rue; elle est insérée entre celles des immeubles déjà existants avec la mention «scission en 2 » en face de l'entrée de chacun des trois édifices de même hauteur (fig. 1). Le deuxième est une vue en plan où on voit que la cour centrale du 20/28 fait écho à celles des projets avoisinants. Même si nous sommes ici face à une volonté de «faire ensemble» qui correspond forcément à un certain degré de «faire pareil», ce cas extrême nous renvoie à la réalité de la production architecturale : l'inscription dans un contexte suppose une sélection dans cette extériorité que constitue

3. Voir à ce sujet Olfa Meziou Baccour, «Étude génétique et architecture. De l'ante-projet à la commande. Les tribulations d'une casa Rustici à Belleville», Littérature, no 178, juin 2015, p. 93-104.

4. Daniel Guibert, Du jeu des références et de leurs valeurs dans la création d'un matériel génératif de la projétation en architecture, rapport constitué dans le cadre d'une consultation restreinte de recherche sur «les processus et les savoirs de la conception architecturale», Paris, Direction de l'architecture et de l'urbanisme, 1991, p. 10.

5. Olfa Meziou Baccour, «Étude génétique et architecture...», art. cit., p. $97-98$. 


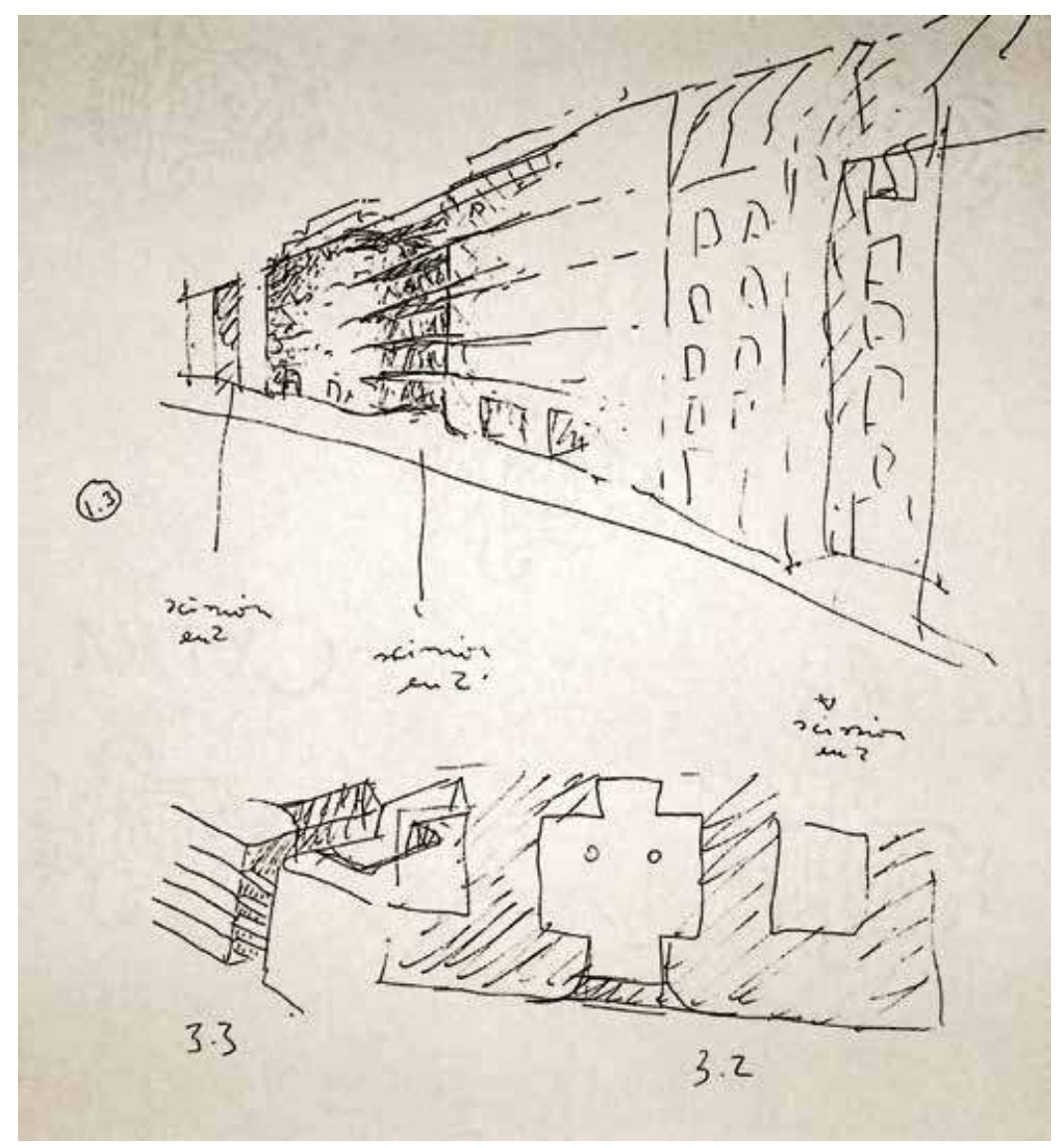

Fig. 1 : D3. Vue en perspective de la façade sur rue. Esquisse d'un plan et d'une axonométrie. Les trois dessins sont réalisés au stylo sur papier pelure de dimension environ A4. 6 septembre 1986.

(C) F. Montès

l'environnement physique immédiat. On voit apparaître une sorte d'extériorité inhérente au projet, notion paradoxale qui viendrait s'opposer à une extériorité «étrangère » dont la convocation - et l'intégration - relèverait des désirs et des doctrines de l'auteur et qui serait l'objet de l'enquête exogénétique. En tout état de cause, que ce soit pour une extériorité inhérente ou une extériorité convoquée, les prélèvements effectués par l'architecte donnent forme à l'extériorité et à l'œuvre en cours. L'exogenèse en constitue la trace et permet donc de les identifier :

Bref, l'exogenèse ne désigne pas les «sources» de l'œuvre (telle personne réelle, tel lieu, telle ouvre littéraire, etc.), mais la trace repérable de ces référents-sources en termes de documents (écrits ou transposés) qui se trouvent attestés dans le dossier de genèse 6 .

La question du repérage des traces est donc au fondement de l'étude exogénétique. Elle entraîne d'autres questions : Comment identifier et déceler la trace? Comment reconnaître le référent exogénétique qui n'est presque jamais représenté comme tel? Comment d'ailleurs imaginer représenter un projet architectural «source»? Par sa façade, son plan, une coupe, une volumétrie, un principe d'organisation? Ainsi, à côté de ce premier enjeu de l'enquête exogénétique, apparaît un second enjeu : quelles formes «prélève» l'architecte dans les références convoquées ${ }^{7}$ ? Quelle sélection opère-t-il? Quelle première transformation, quelle première action endogénétique s'exprime déjà dans la première trace?

6. Pierre-Marc de Biasi, «Qu'est-ce qu'un brouillon?...», art. cit., p. 47-48.

7. Voir à ce sujet Olfa R. Meziou Baccour, «Borrowing modes of patrimonial reference as signifier of architectural form» dans Forms of Patrimony, ELSA, vol. 2; III-IV, 2010, p. 82-94. 


\section{L'enquête. Inférence et interférences des entretiens}

Si la question qui nous importe est l'incidence des entretiens sur la conduite de l'enquête exogénétique, celle-ci est déterminée par l'ensemble du dossier de genèse qui revêt ici une forme particulière puisqu'il a fait l'objet de deux recherches successives et complémentaires : un premier dossier de genèse a été constitué par Pierre-Marc de Biasi entre 1986 et 1990 ; nous l'avons repris et complété entre 2002 et 2003.

Le dossier de genèse, dans sa première version, était constitué de deux grands blocs de brouillons. D'une part, une série de 159 documents datés et une série de 38 documents non datés mais représentant tous la même partie de l'édifice : la façade sur rue en perspective. Il s'agissait de la partie dite «réfection de l'entrée». Fernando Montès avait déposé un premier permis de construire et celui-ci avait été refusé par l'architecte des bâtiments de France (ABF) impliqué du fait de la proximité de la bouche de métro de la station «Couronnes » classée «monument historique». L'ABF avait jugé la façade trop monumentale par rapport à la petite rue Ramponneau et Fernando Montès avait été prié de réduire sa taille. Les deux dossiers de permis de construire étaient manquants mais une série de photographies tant de la maquette définitive que de l'édifice en cours de construction puis construit était disponible. Les photographies de la plupart des 159 premiers documents étaient également à disposition.

Par ailleurs, deux parties des entretiens avaient été retranscrites mais les bandes sonores sur lesquelles étaient enregistrées vingt-cinq autres heures restaient introuvables. Deux photographies de la casa Rustici de Giuseppe Terragni étaient accompagnées d'un article en italien sur ce même projet.

On y trouvait aussi un document que l'on pourrait qualifier de «post-génétique»: il représente un projet de villa-atelier Le Corbusier à Cachaga au Chili (voir fig. 10) où Fernando Montès réutilise l'élément vertical auquel il a dû renoncer lors de l'étape «réfection de l'entrée».

À côté de cela, il y avait le rapport - cité plus haut - où étaient consignés les résultats de l'étude menée par PierreMarc de Biasi et Fernando Montès, le dispositif d'analyse imaginé et son application sur les onze premiers dessins.
La prise de contact avec Fernando Montès s'est faite le jour de la visite de l'édifice, son agence se trouvant alors depuis la construction de l'immeuble - au rez-de-chaussée du 20/28 de la rue Ramponneau. Il s'agit d'une visite assez brève, circonscrite au vide intérieur de l'édifice. L'architecte explique, au cours de la discussion, que «normalement ça devrait communiquer avec les autres immeubles mais peut-être un jour...». Il se lance ensuite dans une explication sur la genèse des différents blocs, en particulier celle du bloc $\mathrm{C}$ : «Ils sont tous différents, vous savez...» Je ne dis rien. Tous les blocs semblent identiques.

À ce stade, il y a déjà un ensemble d'indices qui permettent d'identifier de l'exogenèse, c'est-à-dire les traces repérables de référents-sources :

1. La casa Rustici de Giuseppe Terragni ${ }^{8}$. Une déclaration de Fernando Montès retranscrite dans les entretiens : «Alors moi, depuis longtemps, je voulais faire une casa Rustici à Paris ${ }^{9}{ }$, les deux photographies de l'œuvre de Giuseppe Terragni - elles avaient été placardées à l'agence - et enfin le premier dessin du projet (D1, fig. 3) représentant la façade sur rue, en continuité avec les immeubles avoisinants, une façade où «la symétrie produit du double, et où les liens qui forment l'entité sont de plus en plus ténus, et de plus en plus abstraits [...] [et] se résument à

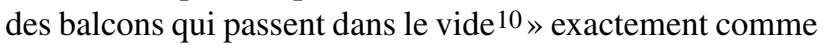
dans la description que fait Fernando Montès de la casa Rustici. Dès le premier dessin, le projet comporte donc la trace repérable de ce référent source : la casa Rustici.

2. Dans le dessin D10, un nom, «Ginsberg», écrit en toutes lettres. Il s'agit de Jean Ginsberg, architecte français d'origine polonaise connu pour être une figure de l'architecture de l'entre-deux-guerres. Pierre-Marc de Biasi évoque, dans son rapport (déjà cité), «la reprise de l'initiale G de Ginsberg pour dessiner la forme de la découpe de la peau devant le noyau arrondi ». Ce dessin fera la couverture du numéro «Architecture» de la revue Genesis ${ }^{11}$.

8. Giuseppe Terragni, célèbre architecte italien de l'époque fasciste, avait conçu avec Pietro Lingeri à Milan en 1933-1935 un bâtiment moderniste (La casa Rustici) qui est resté le symbole du «rationalisme italien» : un immeuble résidentiel de 6 étages bâti pour le compte de Vittorio Rustici. 9. Entretiens Pierre-Marc de Biasi / Fernando Montès (1986-1990).

10. Ibid.

11. Genesis, no 14, 2000, coordonné par P.-M. de Biasi et R. Legault, en ligne sur persee.fr. 


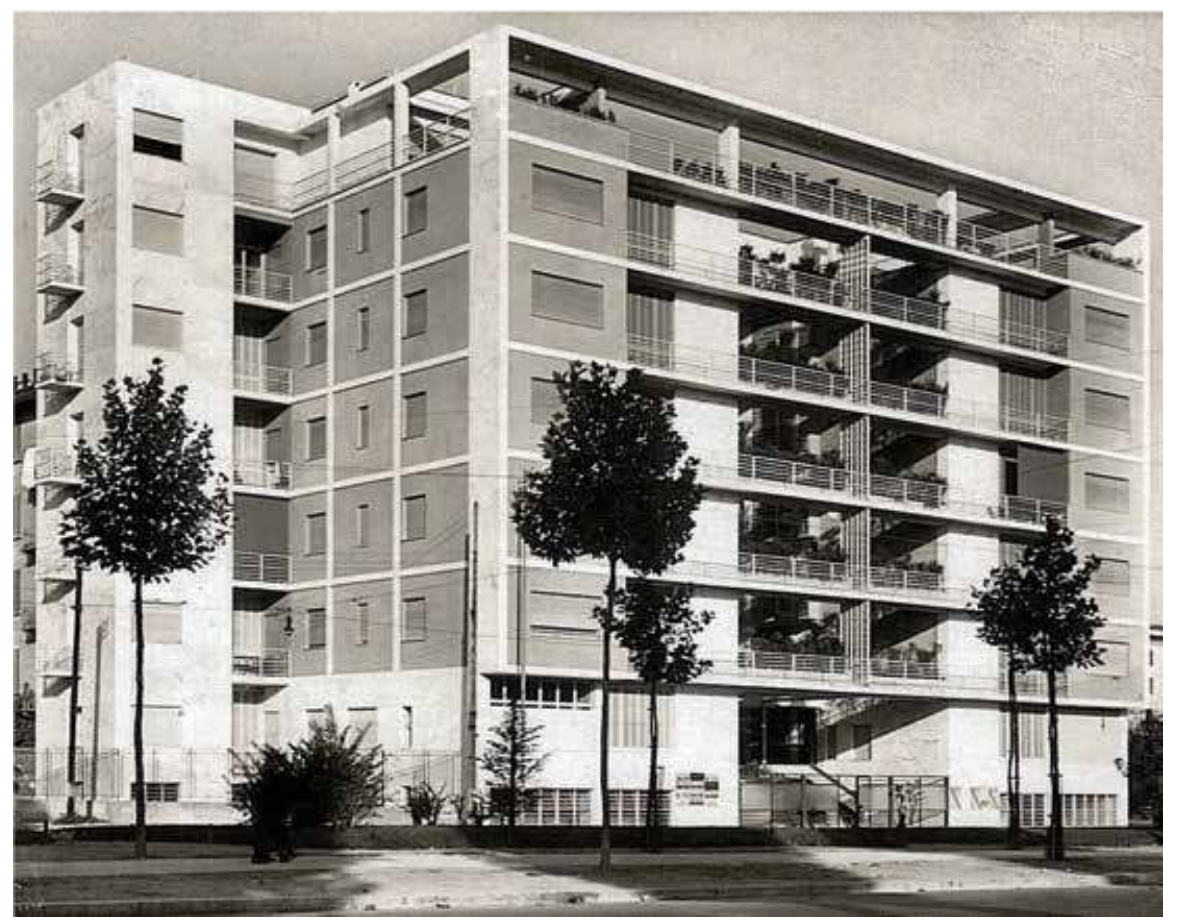

Fig. 2 : Milan, Corso Sempione, casa Rustici, Giuseppe Terragni (1935).

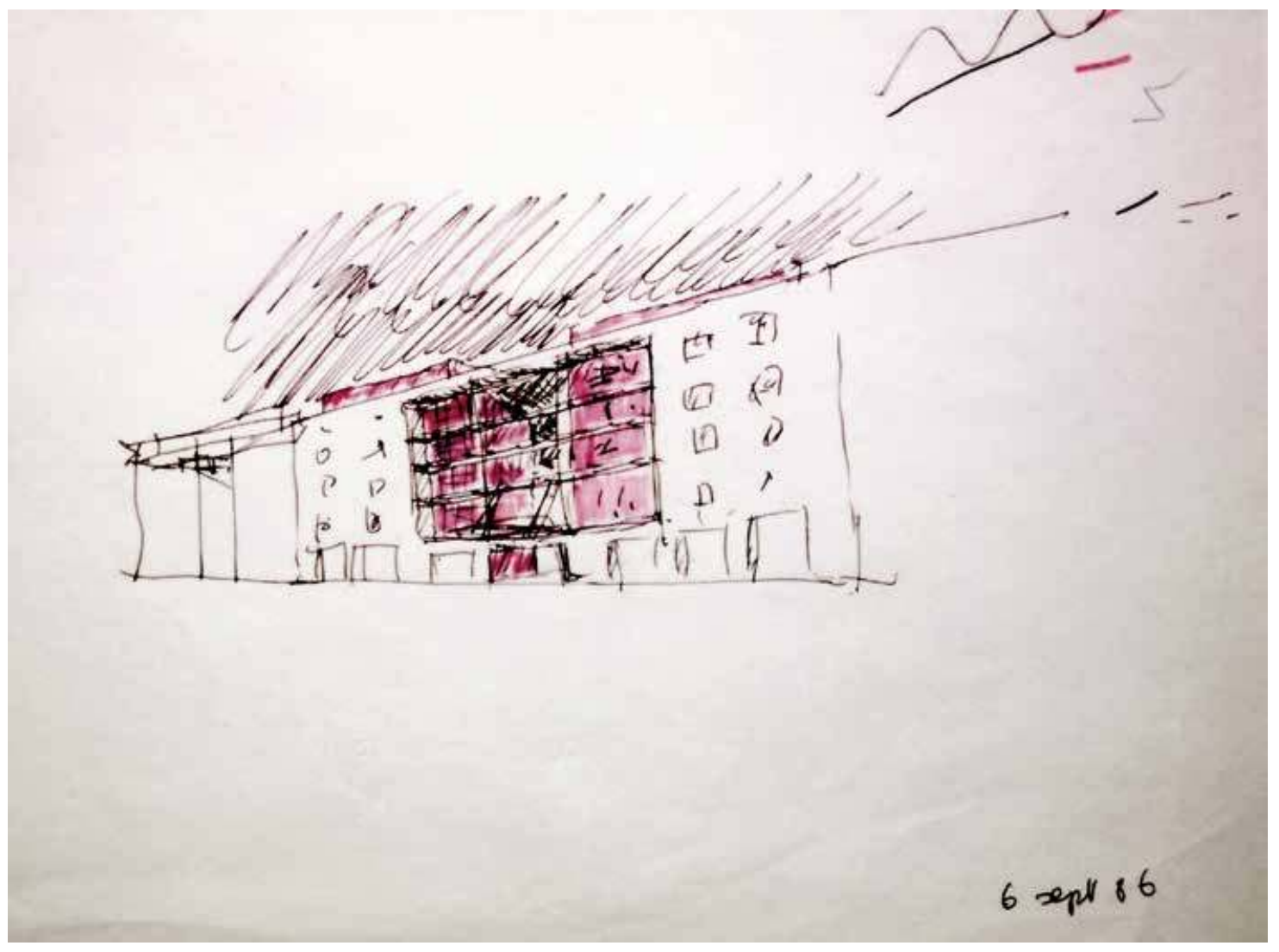

Fig. 3 : D1. Premier dessin du projet. Vue en perspective de la façade sur rue.

Réalisé à main levée sur papier pelure. Stylo et crayon de couleur. Format A4. 6 septembre 1986. (c) F. Montès 
3. Dans le dessin D50 apparaissent deux autres noms : Sautner et Tschumi. L'ante-projet du 20/28 rue Ramponneau comporte à la fin, après que tout a été relativement abouti, une série de dessins insolites ou plutôt génétiquement anachroniques. En effet, alors que les dessins s'apparentaient plus à des dessins d'APD (avant-projet détaillé), alors que le projet semblait avoir atteint un certain niveau de stabilité (on trouve sur le document D145 une coupe axonométrique le long de la faille, une façade parfaitement calée au dénivelé de la rue avec quatre coupes mesurées correspondant à différents points du dénivelé), arrive une série de dessins concernant un des blocs arrière, le bloc $\mathrm{C}$, où la recherche de la forme repart comme à zéro. Le bloc $\mathrm{C}$ devient un cube que Montès joue à sculpter et resculpter comme pour explorer son potentiel plastique. Difficile de ne pas penser aux vingt-cinq folies de Bernard Tschumi, dont le nom est inscrit une centaine de documents plus tôt (D 50), toutes conçues sur le principe de déclinaison d'un cube de 10,8 m de côté. L'emprunt aussi était intéressant. Ni image, ni géométrie, ni spatialité mais une façon de procéder pour générer de la forme.

Voyons maintenant ce qui arrive après l'enrichissement du dossier de genèse, notamment grâce à de nouveaux entretiens.

1. Pour la casa Rustici, les nouveaux entretiens n'apportent rien mais les brouillons du projet, si. Ils montrent que cette façade double ${ }^{12}$ reliée par des balcons aériens est peutêtre l'arbre qui cache la forêt. La casa Rustici apparaît en effet comme un élément globalement structurant, bien plus qu'une façade symétrique qui joint deux villas siamoises par une peau constituée de balcons aériens «sublimes 13 ». La symétrie - plutôt dissymétrie quand on regarde les plans - a été abandonnée au profit du continuum spatial organisé, de cette coulée intérieure qui devait traverser tout l'îlot mais qui ne sera effective que «peut-être un jour...» comme le dit Montès. De plus, comme les projets avoisinants sont situés sur les côtés du 20/28 mais aussi à l'arrière, la faille centrale (coulée principale parallèle à la rue Ramponneau) a dû croiser une autre faille perpendiculaire (et décalée par rapport au milieu du terrain). Au lieu de deux blocs, ce seront donc quatre blocs que le projet comportera : une strate sur rue constituée par les blocs A et $\mathrm{B}$, et une strate arrière constituée par les blocs $\mathrm{C}$ et $\mathrm{D}$. Quatre blocs dont les emprises au sol, à chaque fois différentes, seront déterminées par le tracé des failles qui prolongent les failles des voisins. Quatre villas donc, pas tout à fait siamoises (fig. 4).

Par ailleurs, une des premières caractéristiques relevées par Fernando Montès dans la casa Rustici est que celle-ci comporte un corps et une peau. Dès le premier dessin et au fil de l'ante-projet, l'architecte maintient cette opposition corps/peau ou noyau/peau en utilisant notamment la couleur, comme l'a fait Giuseppe Terragni dans son projet - Montès utilise d'ailleurs les mêmes couleurs que Terragni dans son projet final. Chacune des deux strates $(\mathrm{AB}$ et $\mathrm{CD})$ sera traitée comme deux noyaux reliés à l'avant comme à l'arrière par une peau essentiellement constituée de balcons. Mais Montès va plus loin. Tout se passe en effet comme s'il dépassait la binarité de cette opposition pour créer différentes classes d'objets soulignées par l'usage de la couleur. C'est ainsi qu'on verra apparaitre, dès le document D54 (fig. 5), en plus des noyaux (en jaune) et des peaux (en bleu), les longs objets verticaux (le totem et les cages d'escalier en rouge) mais aussi le soubassement (en vert) qui pénètre dans la faille et deviendra un prolongement intérieur de la rue (D136, fig. 6) concrétisant ainsi cette idée de prolongement de l'espace public primaire à l'intérieur de l'immeuble adoptée par l'équipe Duhart, Borel, Ripault et Montès. Cette dernière configuration était aussi inscrite dans la casa Rustici. Monique Eleb, citée plus haut, parle d'un «bâtiment organisé autour d'une cour surélevée par rapport au niveau

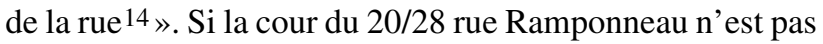
surélevée, on voit dans les dessins D100 et suivants que Fernando Montès explore l'idée de prévoir des parties surélevées qui seraient plus privées que les parties inférieures, une façon d'expérimenter les formes de l'autre principe cher au groupe : le continuum spatial organisé, pouvoir passer du public au privé en camaïeu par une succession d'espaces

12. La casa Rustici, le référent-source est composé de deux façades identiques pour deux édifices distincts et qui ne sont relié(e) s que par des balcons. Montès va dédoubler ce lien, deux fois, au niveau de chacune des deux strates.

13. On notera que pour Monique Eleb, la casa Rustici évoque tout autre chose : «Un bâtiment en "U" refermé aux niveaux supérieurs par un bandeau de balcons qui laissent filtrer la lumière, bâtiment organisé autour d'une cour surélevée par rapport au niveau de la rue. » Voir Monique Eleb, Anne-Marie Châtelet, Jean-Claude Garcias et al., L'Habitation en projets; de la France à l'Europe : Europan 1989, Liège, Pierre Mardaga, 1990.

14. Monique Eleb et al., L'Habitation en projets..., op. cit. 


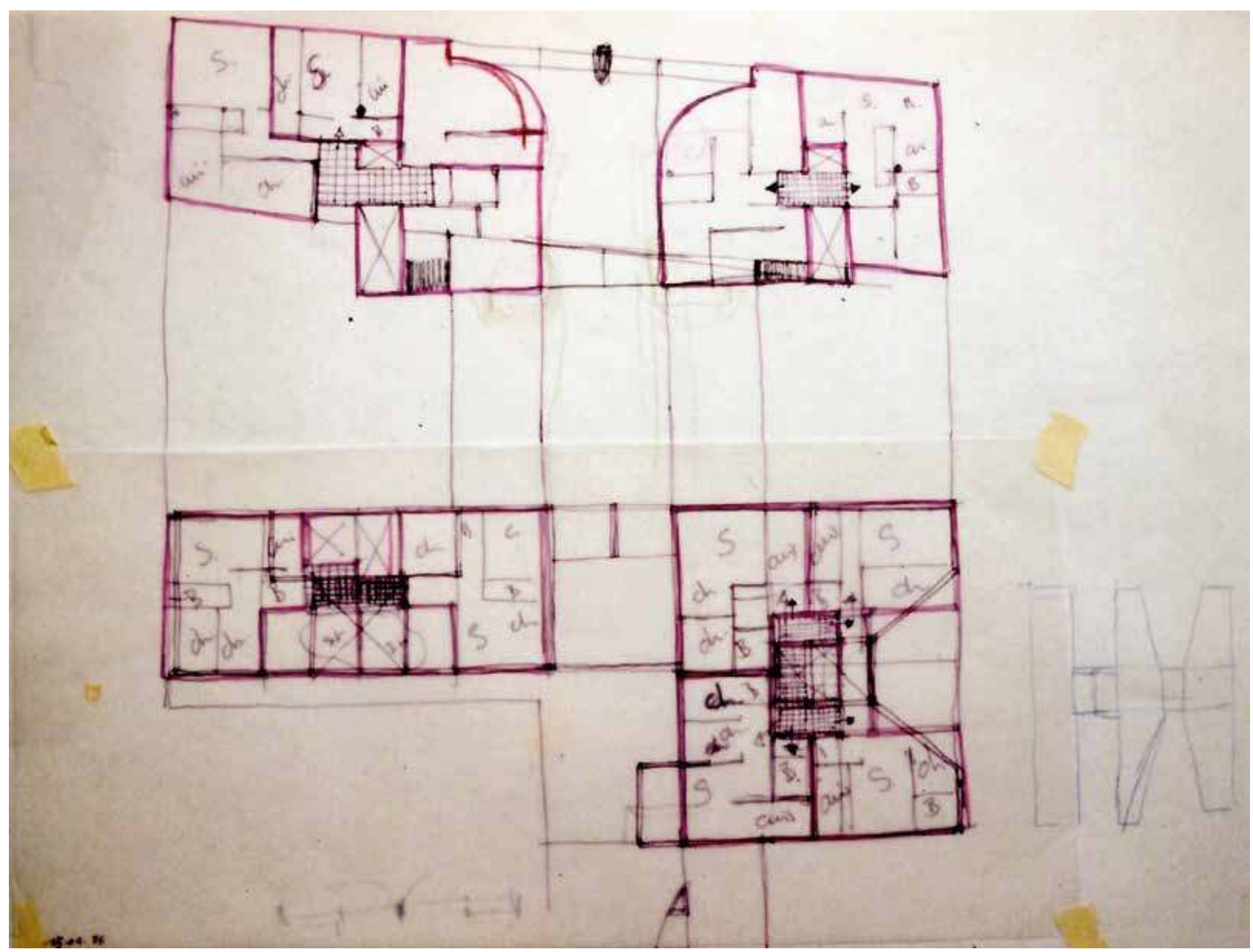

Fig. 4 : D16. Vue en plan du projet. Document constitué de deux calques :

calque correctif du 15 septembre 1986 sur calque du 12 septembre $1986.31 \times 24 \mathrm{~cm}$. Les quatre blocs ne sont plus identiques. (C) F. Montès

de moins en moins publics. Ce dispositif, la cour surélevée, a été ensuite abandonné.

2. Pour le dessin D10 avec le nom de Ginsberg écrit en toutes lettres, nous avons réinterrogé l'architecte. Pas de réponse mais un grand discours sur l'architecture de l'entre-deux-guerres et ses «héros »: Jean Ginsberg et son immeuble de l'avenue de Versailles (1930-1931, en collaboration avec Berthold Lubetkin), Bruno Elkouken et ses ateliers pour artistes du boulevard Raspail (1934), Pierre Chareau et sa maison de verre de la rue Saint-Guillaume (1928, en collaboration avec Bernard Bijvoet). Nous nous sommes rendus sur place. Il se peut que ce jeu sur l'initiale évoqué par de Biasi ait eu lieu mais l'immeuble de Ginsberg et Lubetkin au 25 avenue de Versailles comporte non seulement le même arrondi au niveau de l'entrée mais aussi une façade dont le traitement des ouvertures sera utilisé dans les dessins D43 et D72 pour explorer les possibilités de traitement d'une des façades intérieures du projet (celle du bloc D).

Les briques de verre de la célèbre façade de la Maison de verre, quant à elles, apparaissent de manière très claire dans le traitement des cours circulaires (fig. 7, D69) qui structurent le projet mais disparaissent pour des raisons de coûts. On découvre entretemps que Fernando Montès a écrit un article dans Global Architecture (no 46) en 1977 intitulé «La Maison de verre».

Enfin, on retrouve les bow-windows entièrement en verre de l'immeuble d'Elkouken du 216 boulevard Raspail mais également une même position dans la composition de la façade dans les esquisses pour le bloc D du dessin D42. 


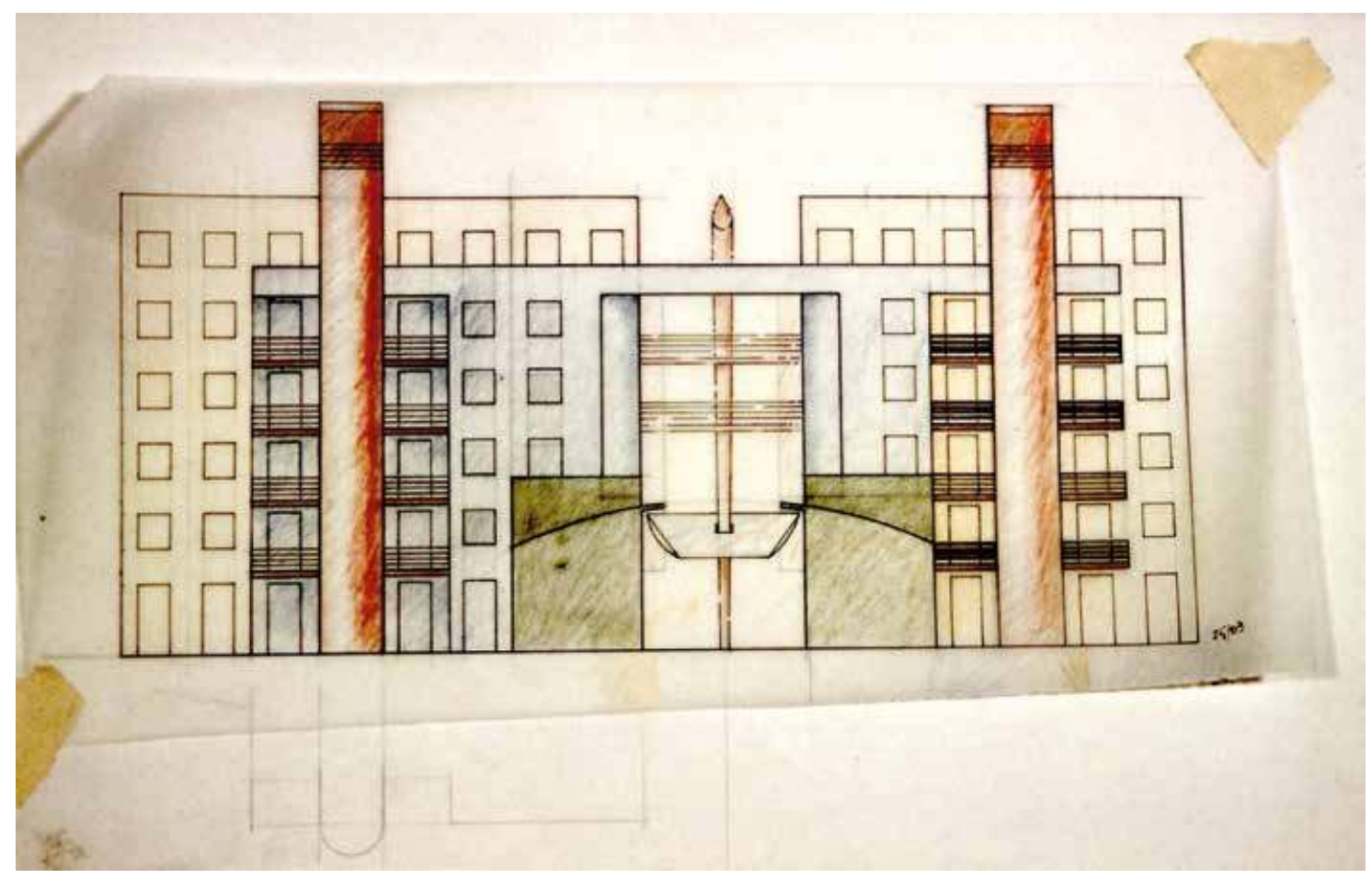

Fig. 5 : D54. Façade arrière des blocs A et B. Document composé de deux calques superposés. Dessins au tire-ligne, mine de plomb et crayons de couleur. 25 septembre 1986.

(C) F. Montès

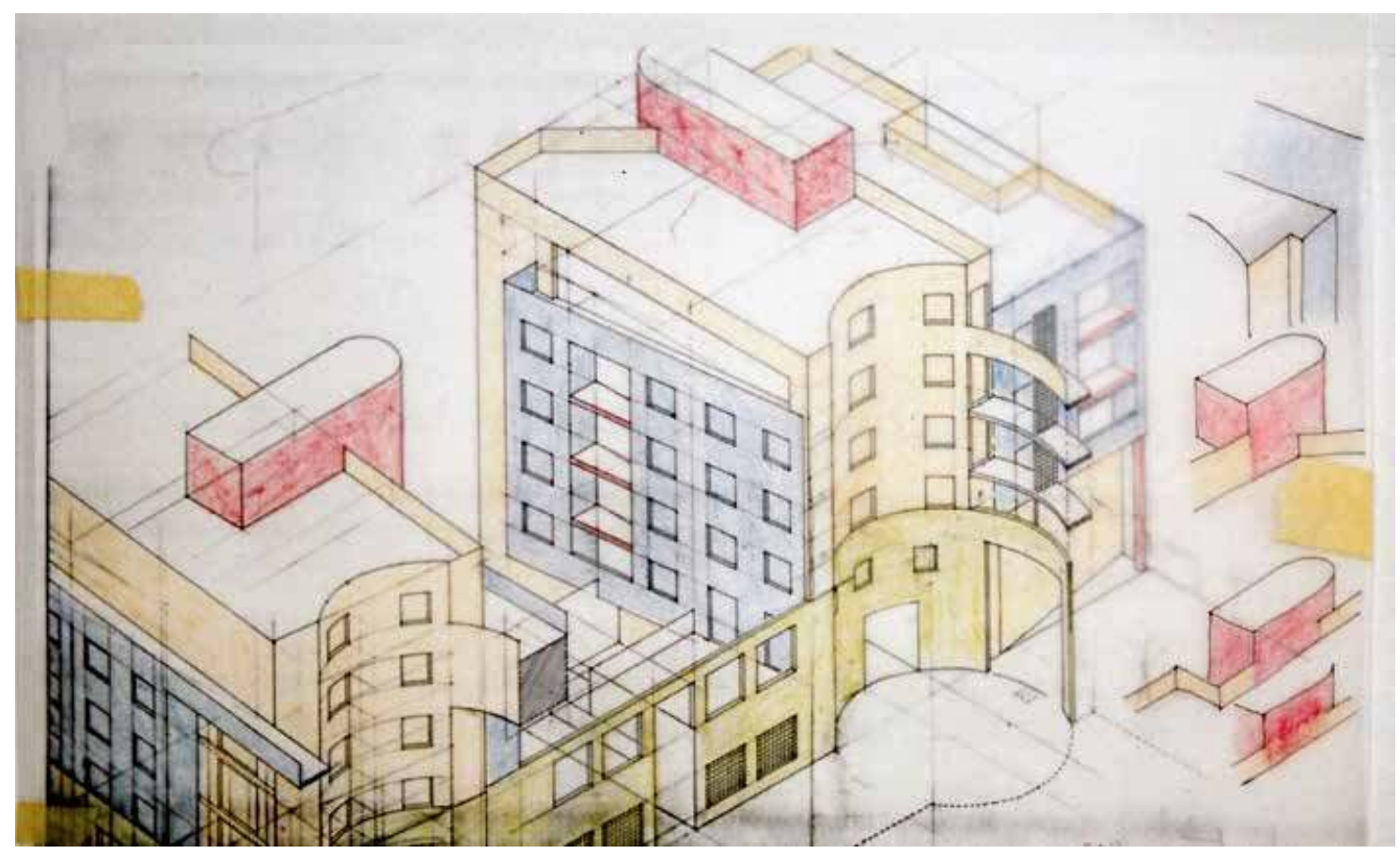

Fig. 6 : D136. Coupe axonométrique le long de la faille (blocs B et C) et petits dessins pour le haut de la cage d'escalier du bloc C. Document composé d'un calque superposé à une photocopie. Dessin au tire-ligne et crayons de couleur. 9 novembre 1986. 


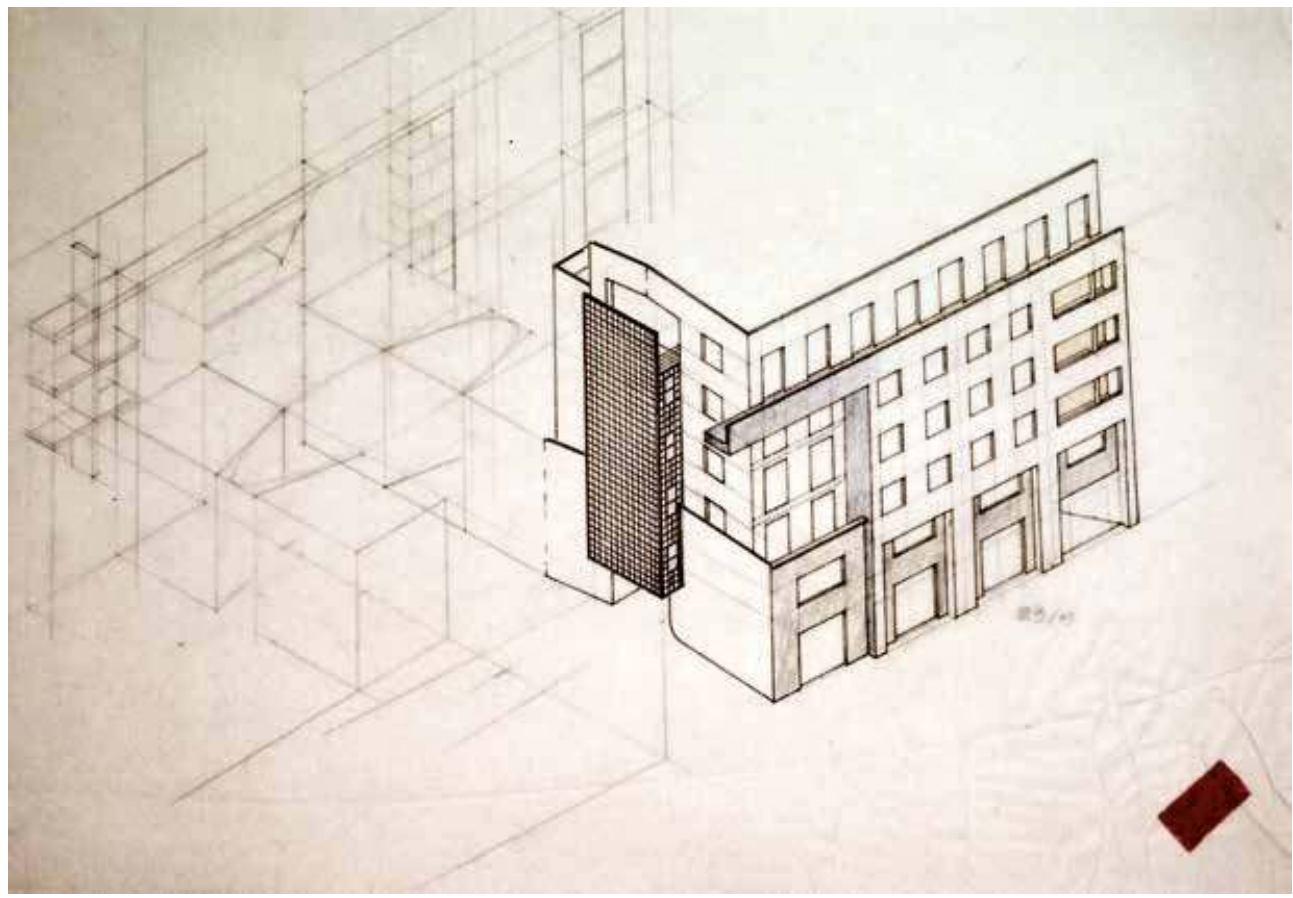

Fig. 7 : D69. Vue axonométrique du bloc A et lignes de construction pour l'arrière du projet. Dessin sur calque au crayon, tire-ligne et crayons de couleur. 29 septembre 1986.

(C) F. Montès

Au-delà du jeu plastique de la forme du G, le mot Ginsberg a ouvert des pistes d'investigation et agi ici comme le révélateur d'un monde : une certaine architecture de l'entre-deux-guerres dont les traces tendront à s'effacer dans la version finale du projet. L'apport des entretiens a été ici très important puisqu'il a permis de retrouver les projets-sources et d'identifier leurs traces dans l'ante-projet.

3 . En ce qui concerne le troisième indice, nous avons aussi posé la question et exposé notre hypothèse concernant le lien entre Tschumi (+ Sautner) et les derniers dessins. Montès ne reconnaît pas cette «source». Pour justifier la présence des deux noms sur le document D50, il explique qu'il ne s'agit que d'un griffonnage; il devait être en train de répondre au téléphone à Tschumi qui, ayant gagné le concours de la Villette, l'aurait sollicité pour l'aider à choisir un entrepreneur, et Montès lui aurait recommandé Sautner. Pour expliquer ce «jeu» sur le bloc C, parce que c'est de lui qu'il s'agit, Montès explique qu'il a fait l'objet d'une recherche sur une composition volumétrique autour d'un axe vertical (la cage d'escalier) évoquant une référence pas tout à fait architecturale : le chawarma, ce sandwich libanais à base de viande dont les tranches ont été empilées autour d'une tige métallique verticale.

4. Au cours des entretiens effectués en juin 2003 (deuxième série d'entretiens), Fernando Montès parle d'une place qui l'aurait autant marqué que la casa Rustici de Giuseppe Terragni : la place Saint-Pierre de Vigevano, qui fait partie, pour lui, du patrimoine architectural et urbain mondial et qui constitue le modèle de «la place française au meilleur moment». Conçue vraisemblablement - mais sans signature - par Léonard de Vinci, Bramante et un architecte moins connu mais désigné par le duc de Vigevano : Andrea Colonna, la place, à l'instar de la place Vendôme à Paris, est conçue comme « un trou qui est fait dans un solide [...] alors que presque toutes les places italiennes sont conçues comme des collections de monuments différents $15_{\text {». }}$.

À l'époque où Vigevano, capitale de la machine à faire la chaussure, devient très riche et très active, son duc «n'admettra plus que cette ville soit un tel labyrinthe et décidera de créer un lieu de rencontre des gens, un lieu qui soit bien clair [...]. Qu'est-ce qu'il va faire? Il va détruire beaucoup de maisons pour faire un ovale. Et la place est un ovale. Et Bramante va projeter une façade qui est exactement la même des deux côtés, comme la place Vendôme... Les maisons ne sont pas les mêmes derrière mais la façade est unificatrice ${ }^{16} . »$

15. Propos recueillis au cours des entretiens en juin 2003. 16. Ibid. 
Fernando Montès, qui a vite fait de structurer son projet en quatre blocs, se trouve au bout de trois semaines dans l'obligation de laisser une partie de son travail en suspens. En effet, les retraits qu'il va devoir observer sur un des blocs (le bloc $\mathrm{C}$, encore lui) sont en négociation. Il doit donc avancer dans le projet tout en laissant de côté une de ses parties. Pour surmonter cet obstacle, il va convoquer la référence italienne et emprunter à la place Saint-Pierre de Vigevano son statut de place symétrique à façade unificatrice, autonome et indépendante de ce qui se passe derrière ses façades. Sur le dessin no 69 (fig. 7), il dessine une place ovale - comme celle de Vigevano - avant de se défaire de la figure géométrique, pour ne conserver que des attributs fonctionnels et organisationnels. La place a fonctionné comme lieu à la fois articulant et possédant une certaine autonomie.

À ce stade, c'est l'entretien avec Montès et sa description de la place Saint-Pierre de Vigevano qui permettent de faire de cet ovale du dessin no 69 un indice exogénétique et qui rendent la référence à la place Saint-Pierre de Vigevano repérable dans l'ante-projet.

Attardons-nous à présent sur la place Saint-Pierre à Vigevano. Elle n'existe pas. Il n'y a pas de place Saint-Pierre à Vigevano. Il y a bien la place Ducale, «commandée» par le duc de Milan, Ludovic Sforza, vraisemblablement dessinée par Donato Bramante et Léonard De Vinci en 1492, considérée comme un des premiers exemples de places de la Renaissance réalisées sur le modèle de forum romain, caractéristique des places lombardes du xve siècle. Autre petite inexactitude : la piazza Ducale n'est pas ovale. C'est une place rectangulaire de $134 \mathrm{~m}$ de longueur sur $48 \mathrm{~m}$ de largeur, construite sur trois côtés, le quatrième étant occupé par la cathédrale, il Duomo, dont la façade concave de style baroque est le seul élément en rapport avec la forme ovale supposée. Par ailleurs, il existe bien une place ovale en Italie, elle se trouve en Toscane; c'est la place de l'Amphithéâtre (piazza dell'Anfiteatro) de la ville de Lucques (Lucca), édifiée au Moyen-Âge sur les restes d'un amphithéâtre romain.

Dans ce cas de figure, si Fernando Montès n'avait fait que parler de la place de Vigevano sans évoquer ce faux attribut (sa forme ovale), la référence à cette place n'aurait pas pu être attestée sur le dessin no 69. Dans ce cas, quelle est finalement la référence convoquée ? La place Ducale de
Vigevano, la place de l'Amphithéâtre de Lucques ou une référence hybride imaginaire qu' aurait rapportée Fernando Montès d'un grand voyage (non attesté) à travers l'Italie ?

\section{Le cas Mallet-Stevens. À l'origine, un mot : «totem»}

Dès le quatrième document (D4), daté comme le premier du 6 septembre 1986, l'auvent qui marquait la scission en deux de la façade se transforme en une «colonne : une barre verticale nettement posée en noir au centre du diaphragme d'ouverture de la façade, avec un auvent qui prend la forme d'une section d'aile d'avion ${ }^{17}$.» C'est la première manifestation d'un élément que Fernando Montès désignera par le mot «totem». Interrogé une première fois sur l'origine de cet élément, il parlera de «choses mystérieuses» qu'il ne peut pas ou ne veut pas rendre plus explicites. Ce totem est un élément important du projet auquel, cependant, Montès devra renoncer après le premier dépôt de permis de construire, l'architecte des Bâtiments de France, comme décrit plus haut, l'ayant jugé monumental. Présenté comme «écho de la liberté intérieure du bâtiment», signalant une sorte d'alcôve publique un peu comme le ferait un auvent pour les passages couverts parisiens dont Montès dit s'être inspiré, c'est ce totem qui nous conduira à l'architecte Rob Mallet-Stevens.

Dans les entretiens réalisés avec Pierre-Marc de Biasi entre 1986 et 1990, on trouve bien une allusion à Rob Mallet-Stevens : Fernando Montès rend hommage non pas à l'architecte mais au scénographe (décorateur de film). Il ne l'évoque d'ailleurs pas pour citer une de ses œuvres en référence, comme la casa Rustici de Terragni ou la place de Vigevano, mais lors d'une conversation sur l'art, pour dire que les décors qu'il a réalisés pour le cinéma et notamment pour le film L'Inhumaine (sorti en 1924) ont peut-être eu plus d'impact, sur le plan culturel, que ses œuvres architecturales.

17. Pierre-Marc de Biasi, Fernando Montès, Pour une analyse génétique des techniques de projet et du processus de conception en architecture (Étude de genèse des premiers dessins du projet de Fernando Montès, 20-28 rue Ramponneau, Paris, 1986-1990), Paris, Direction de 1'architecture, 1990. 


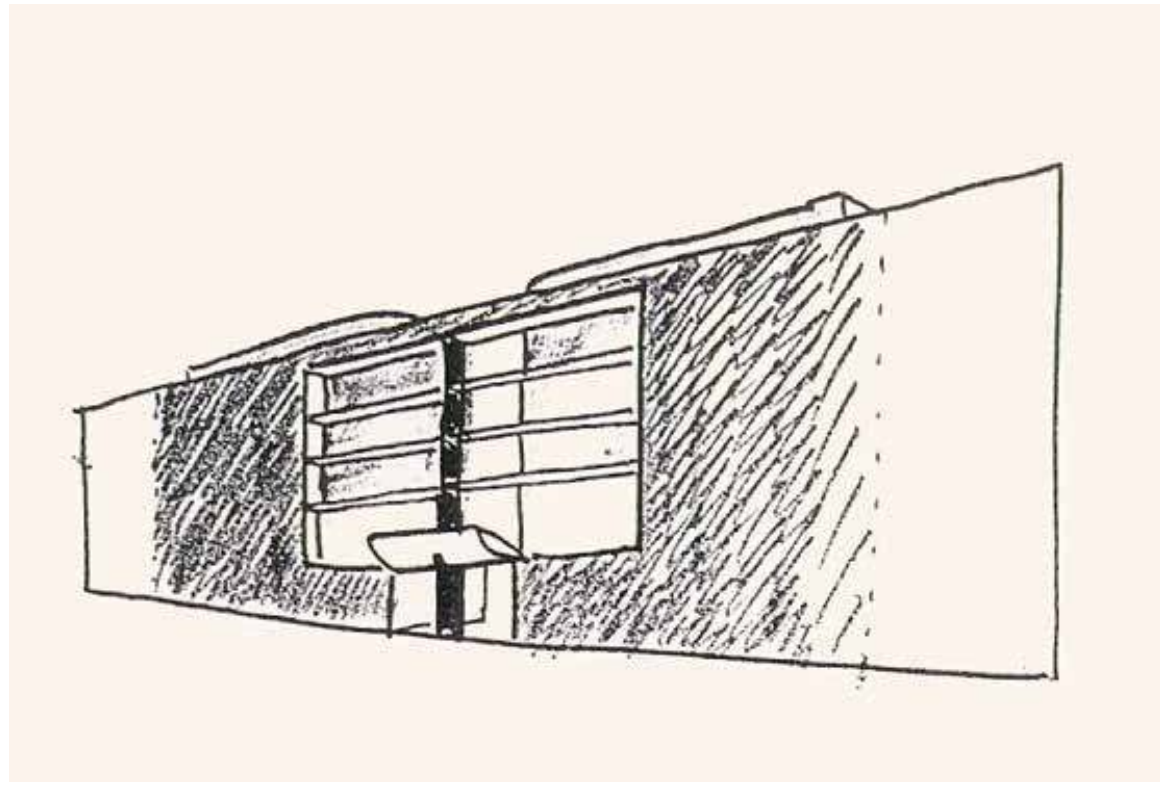

Fig. 8 : D4. Dessin en perspective de la façade sur rue. Dessin au feutre sur papier pelure de format A4. 6 septembre 1986.

(c) F. Montès

Fig. 9 : D7. Vue de face de la façade sur rue. Dessin au stylo et au feutre de couleur sur papier pelure de format A4. 8 septembre 1986.

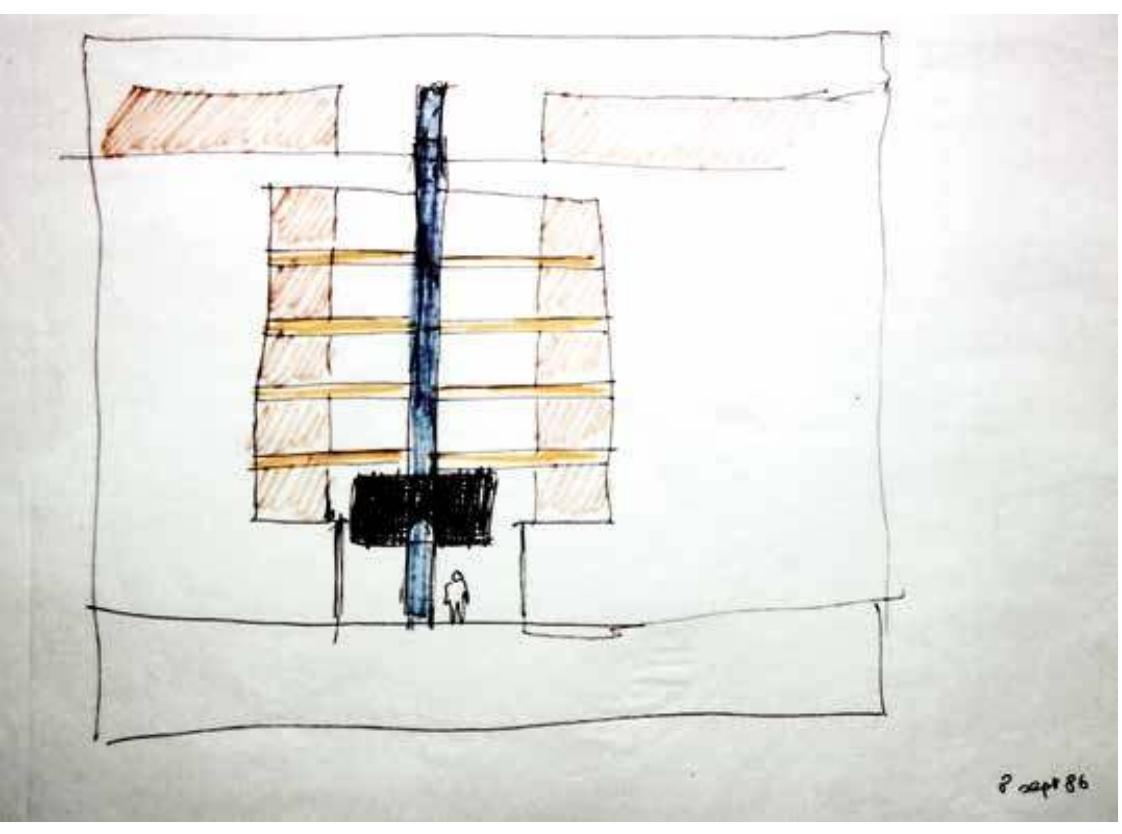

Au cours des nouveaux entretiens réalisés entre 2001 et 2003, Rob Mallet-Stevens réapparaît. Nous avions découvert sur le Net que Pierre-Marc de Biasi avait conçu, à la demande de Fernando Montès, un vitrail qu'ils avaient appelé «totem», pour un nouveau projet parisien élaboré après la conception de l'immeuble de la rue Ramponneau 18 . Voulant comprendre les raisons de cette appellation commune, nous avons posé la question à Fernando Montès. Comme souvent, pas de réponse directe, mais un discours sur les vitraux de Mallet-Stevens et un livre sorti de sa bibliothèque, sur Rob Mallet-Stevens ${ }^{19}$, réalisé, dit-il, avec ses étudiants, où se trouvent les photos desdits vitraux. La consultation de ce livre nous ouvre alors un nouvel univers exogénétique.

18. Il n'y a plus de trace de ce «totem» sur le Net. Par contre, sur le site officiel de Pierre-Marc de Biasi, on trouve à la rubrique «Sculpture / Installations : Commandes publiques - réalisations », un vitrail de $21 \mathrm{~m}$ de hauteur réalisé en collaboration avec Montès en 1993 pour le compte de la RIVP et dont le nom est Géométrie couleurs. Tout porte à croire qu'il s'agit de la même œuvre...

19. Dominique Deshoulières, Hubert Jeanneau, Rob Mallet-Stevens architecte, Bruxelles, Archives d'Architecture Moderne, 1980, 399 p. 


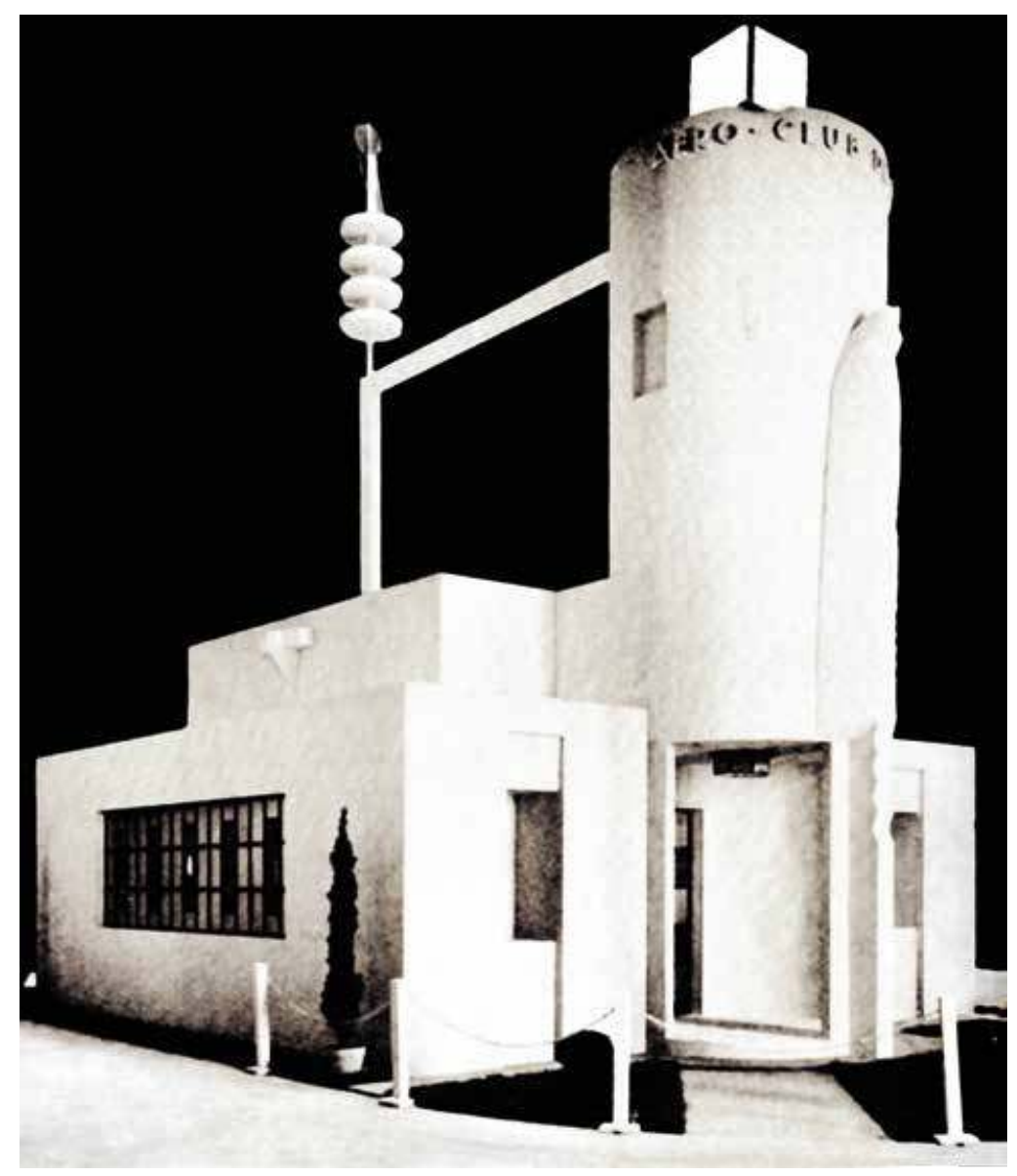

Fig. 10 : Sculpture d'allure totémique d'Henri Laurens adossée au volume cylindrique de l'entrée du pavillon. (C) F. Montès

\section{Le pavillon de l'aéro-club}

Première image, p. 70, dans l'article de Fernando Montès relatif à Rob Mallet-Stevens 20 : la photo de la maquette du pavillon de l'aéro-club présenté par Mallet-Stevens au salon d'automne de 1922. L'entrée de ce pavillon parfaitement symétrique se présente comme un cylindre très haut flanqué de deux baies entre lesquelles, comme collée au volume cylindrique, a été placée une sculpture d'Henri Laurens, ami de Braque et de Picasso. Le totem de Fernando Montès ressemble beaucoup à cette sculpture.

Un autre indice évoqué dans le dossier de genèse initial vient préciser l'élément exogénétique. Lorsque le premier permis de construire a été refusé - justement à cause de ce totem dont les formes n'ont pas plu à l'ABF - l'architecte a réutilisé le dispositif d'entrée conçu pour le projet Ramponneau dans un autre projet : la «maison-atelier» Le Corbusier à Cachagua au Chili. Fernando Montès reprend non seulement la figure du totem avec les balcons très étirés auxquels il a dû renoncer mais aussi l'articulation de cet ensemble à la cour circulaire, qui semblait pourtant être une solution géométrique au problème posé par les différentes données du projet (fig. 11 et 12). L'association totem + cour circulaire - qui n'apparaissait pas dans les premiers dessins du projet Ramponneau - amène à penser que la référence «pavillon de l'aéro-club» a fonctionné dans la genèse du totem mais également dans celle de la cour circulaire.

Ce n'est pas tout : on voit assez vite apparaitre des essais pour trouver une alternative aux sublimes balcons de Terragni, qui prolongent les murs pour traverser le vide. Autour du totem, les balcons prennent la forme d'ailes d'avions pour se détacher des murs de la façade. Dans le dessin D38, la référence au monde aéronautique est plus claire. On trouvera, autour du dessin principal, des petits dessins de manches à air et un dessin d'avion renversé (fig. 13). Apparaît alors un autre mode de référenciation.

20. Fernando Montès, «Case "vitesse" et coin obscur» dans D. Deshoulières, H. Jeanneau, Rob Mallet-Stevens - architecte, op. cit., p. 63-79. 


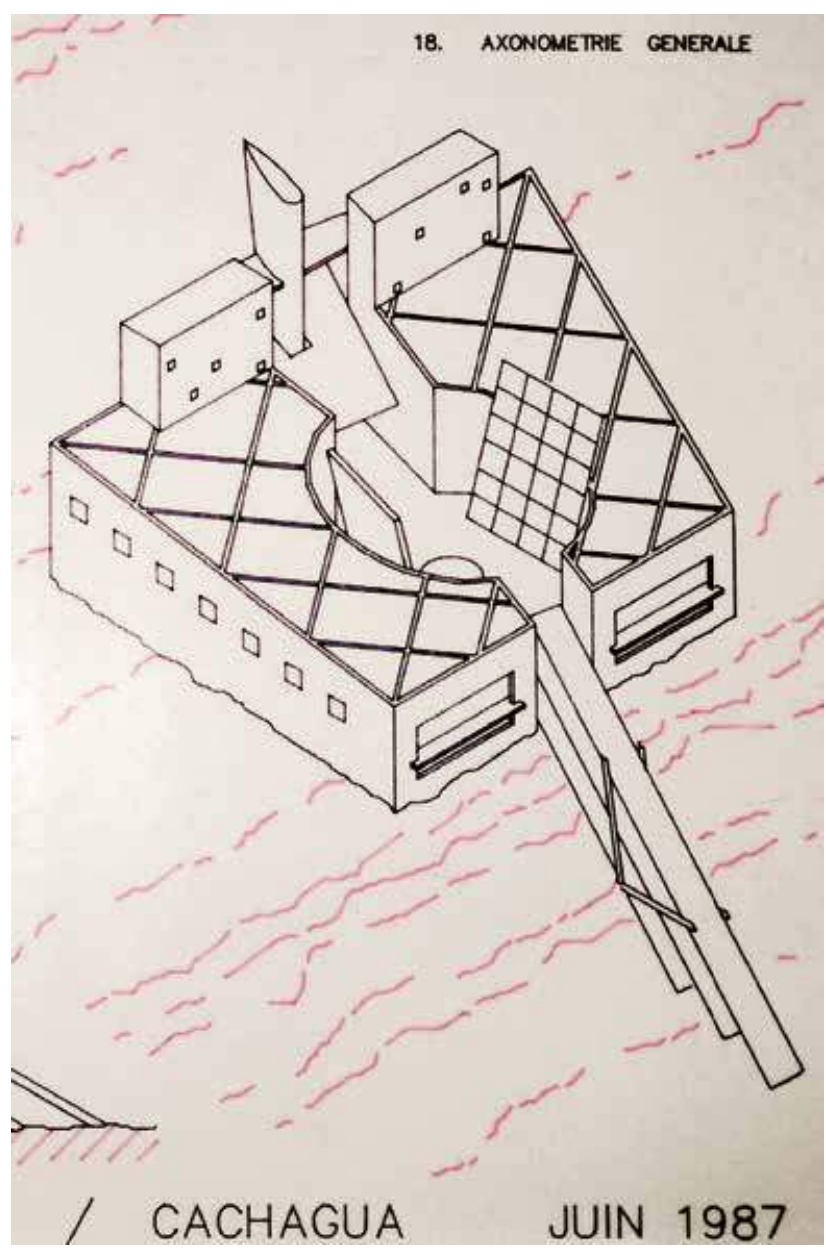

Fig. 11 : Projet de villa-atelier Le Corbusier à Cachaga au Chili. Dessin au tire-ligne et au feutre rouge, 42 x 29,7. Juin 1987.

Fig. 12 : D97. Document composé d'un calque superposé à une photocopie. Dessin au tire-ligne et crayons de couleur (c) F. Montès

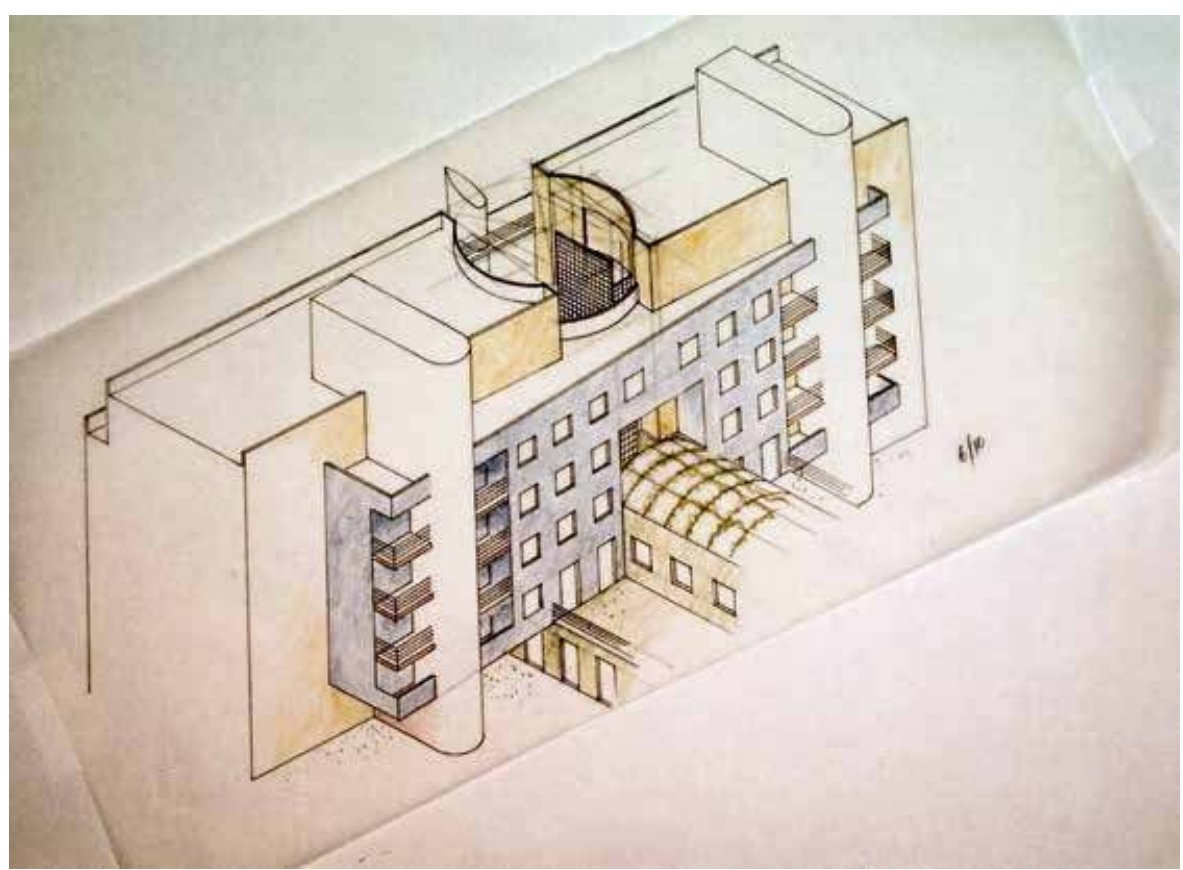




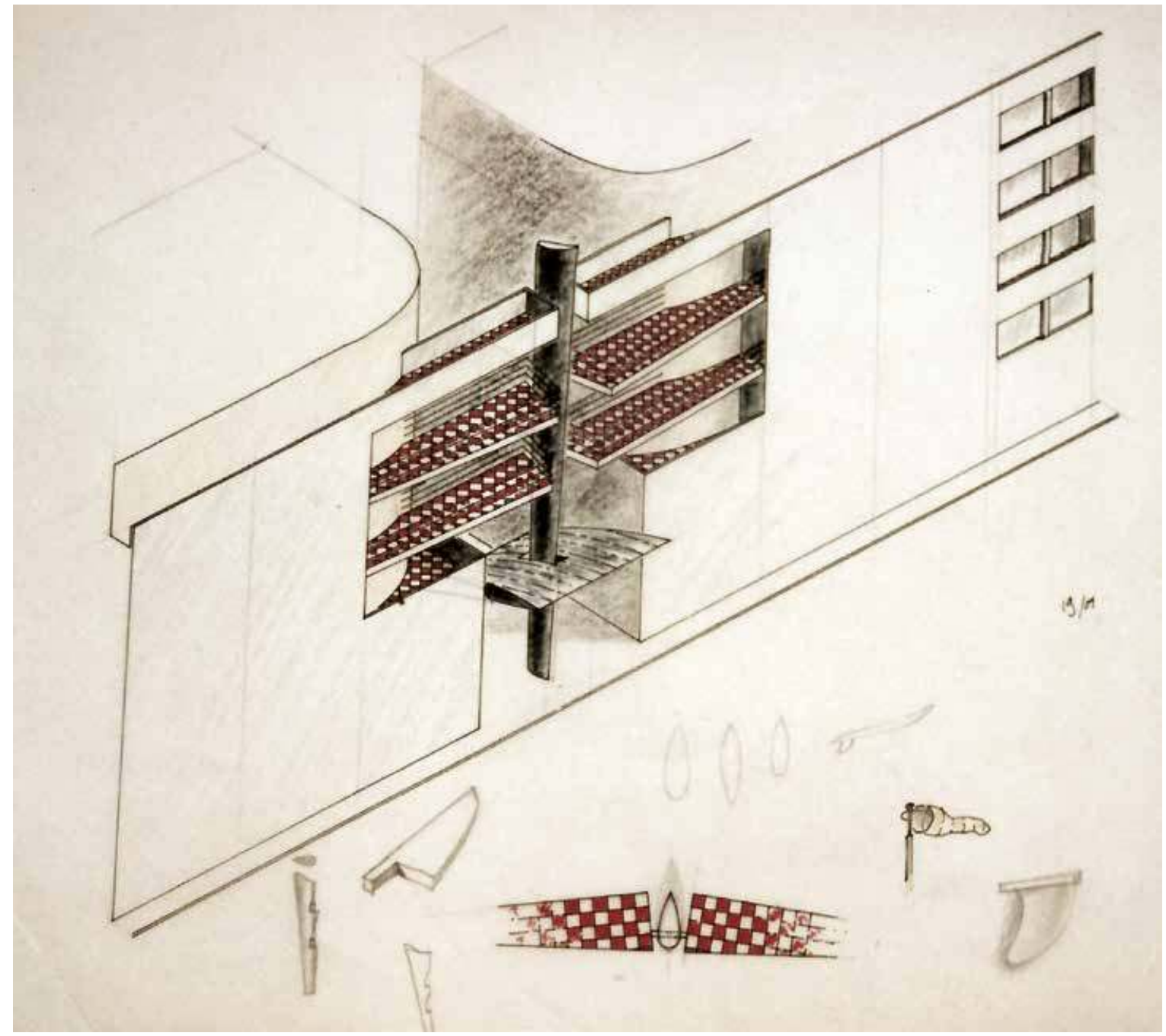

Fig. 13 : D38. Vue en axonométrie de la façade sur rue et des noyaux A et B.

Dessins sur calque légèrement inférieur à A4. Tire-ligne, mine de plomb, stylo, crayon de couleur. 19 septembre 1986.

(c) F. Montès

En effet, la sculpture d'Henri Laurens n'évoque pas le monde aéronautique. Dans son article, Fernando Montès écrit qu'à la limite, «nous pourrions percevoir la sculpture (de Laurens?) surplombant l'entrée, naissant d'un pilier pour se détacher et former une tête, comme une figure de proue, et le bâtiment dans sa totalité comme un navire (aérien?), dont les volumes latéraux formeraient les ailes, et le mât, l'empennage arrière ${ }^{21}$.» Pourtant, pour donner forme à son totem, Fernando Montès semble puiser dans la fonction de ce «temple à l'avion 22 ». On peut l'imaginer pensant à la sculpture d'Henri Laurens, au pavillon de l'aéro-club et au monde de l'aéronautique, monde auquel il n'est pas étranger puisque son père était pilote et son frère fabricant d'avions.
Fernando Montès décrit son totem comme un «écho de la liberté intérieure du bâtiment », comme annonciateur du vide intérieur de l'immeuble. Ces alcôves publiques, espaces collectifs générant des façades en cœur d'îlot, apparentent la casa Rustici, les passages couverts parisiens, les terraces anglais, etc. Ce totem, lui, apparente l'architecture de Rob Mallet Stevens et le monde familial de l'aéronautique. Il apparaît surchargé de significations. En désignant cet objet par le mot «totem», l'architecte était probablement conscient de tout cela, de ce condensé de paternités que ce dispositif portait.

21. Ibid., p. 72.

22. C'est ainsi que Fernando Montès qualifie le pavillon de l'aéro-club. 


\section{Une œuvre, consignée dans un ouvrage}

L'ouvrage consacré à Rob Mallet-Stevens est composé d'une partie de textes - articles et opinions - et d'une autre de 200 pages consacrées aux illustrations. On y trouve les dessins et les photographies de projets d'architecture, d'aménagement, d'objet, de décor filmique réalisés ou non. On s'aperçoit que la plupart de ses projets sont marqués par la co-présence des volumes cylindriques et d'éléments verticaux très évocateurs des mats totémiques souvent placés à l'entrée d'édifices symétriques. L'auvent est aussi très utilisé.

L'étude des documents préparatoires montre que si le générateur primaire du projet est bien la casa Rustici de Giuseppe Terragni, le dispositif d'entrée est, lui, un dispositif d'inspiration malletienne où le pavillon de l'aéro-club est la référence principale mais où l'architecture de Rob MalletStevens en général est convoquée. Cet enchevêtrement des références n'est pas si étonnant dès lors qu'on sait comment Fernando Montès apparente les deux architectes : «MalletStevens, tel un Terragni moins tragique, reste suspendu au-dessus de la frontière, à la limite de l'héroïque et de l'ordinaire 23 . »

En réalité, l'architecture de Rob Mallet-Stevens n'est pas convoquée uniquement pour le dispositif d'entrée. L'observation des illustrations figurant dans l'ouvrage et des documents graphiques de l'ante-projet montre que plusieurs illustrations ont été utilisées en tant que figures, voire en tant qu'allures, comme «point de départ pour réfléchir». Dans la plupart des cas, les solutions préconisées par l'emprunt de ces illustrations ont été abandonnées.

Ainsi, dans la recherche de la forme à donner à la faille au niveau de la strate avant (D12, D35), on retrouve le pavillon d'entrée d'une distillerie à Istanbul24 conçue par Mallet-Stevens. Les balcons étroits qu'il disposera à l'intérieur de la faille à l'angle interne du bloc B (D19) ou, plus tard sur la face contre la faille du bloc C (D39.1), sont identiques à ceux d'une caserne de pompiers se trouvant à Passy 25 (RMS, 1935).

Les différentes variantes qu'il propose comme couronnement aux cages d'escaliers sont toutes celles de Rob Mallet Stevens et enfin le traitement des parois de ces cages d'escaliers par la disposition de fenêtres en longueur ou de grandes ouvertures vitrées se retrouve dans les projets édifiés ou non de Rob Mallet-Stevens.
Et même le bloc C, que Montès affirme avoir conçu comme un chawarma, une composition volumétrique autour d'un axe vertical, apparaît comme un objet malletien. C'est en effet dans un vocabulaire plus architectural mais pour dire la même chose que les deux anciens étudiants de Montès, co-auteurs du livre sur Rob Mallet-Stevens, décrivent l'architecture de ce dernier :

Dans cet «art éminemment orthogonal» selon l'expression de Le Corbusier, les volumes s'organisent autour d'un bloc vertical d'où partent des lignes horizontales résultant de l'utilisation systématique de toitures terrasses et de leurs décrochements successifs. [...]

À toutes les époques de l'histoire de l'art, la maison a été cubique, chaque pays, chaque siècle, chaque mode a marqué son empreinte sur les cubes. [...] Ce ne sont plus quelques moulures gravées dans une façade qui accrochent la lumière, c'est la façade entière. L'architecte sculpte un bloc énorme la maison... l'architecture devient monumentale 26 .

Tout concourt à faire penser que Fernando Montès est complètement imprégné de l'œuvre de Mallet-Stevens. Ayant lui-même dirigé la recherche qui a donné lieu à l'ouvrage, il connaît les projets et leurs images. Il est évident que Montès n'a pas voulu refaire la distillerie d'Istanbul ou la caserne de pompiers de Passy. C'est plutôt la polyvalence du vocabulaire mallétien qu'il semble explorer :

Son vocabulaire, dès 1922 contient déjà tous les éléments. Limité ? Peut-être. Mais étrangement polyvalent. Sans effort apparent, sans rajout ni soustraction, il passe d'une rue à un magasin. $[\ldots]$ «Distancée», avec un dosage très précis entre histoire et abstraction, son architecture ne se confondra jamais avec un jeu de construction 27 .

Comment qualifier la fonction génétique de l'œuvre de Mallet-Stevens? On pourra peut-être parler de matrice de solution. Si la matrice est prise au sens d'un élément qui fournit un appui ou une structure, et qui sert à entourer,

23. Fernando Montès, «Case "vitesse" et coin obscur», art. cit., p. 63.

24. Dominique Deshoulières, Hubert Jeanneau, op. cit., p. 306.

25. Ibid., p. 348.

26. Dominique Deshoulières, Hubert Janneau, «L'exigence de l'architecture» dans Rob Mallet-Stevens, op.cit., p. 23. Cette idée est très visible sur la photo de la villa Martel figurant à la page 268 de l'ouvrage mais aussi dans les dessins de Montès tels que D30 et D149.

27. Fernando Montès, «Case "vitesse" et coin obscur», art. cit., p. 64. 
à reproduire ou à construire, on peut dire que les illustrations $\mathrm{du}$ «Rob Mallet-Stevens » ont servi comme des matrices, des appuis pour aller - selon des trajectoires très diverses chercher des solutions.

\section{Conclusion. \\ Le projet, sériel et spectral}

Dans un texte intitulé «Compétence projectuelle» publié sur son blog le 25 mai 2018, l'architecte Henri Ciriani revient après trente-deux ans d'enseignement sur l'exercice projectuel :

Qu'est-ce que projeter, être compétent au projet? [...] Il s'agit d'être habité par un désir double : primo : tout connaître, par la pensée et par la vue, de l'art de construire; secundo : ne pas se satisfaire de reproduire ou de falsifier la reproduction, mais de transformer l'espace du projet comme s'il faisait partie d'une série, qu'il en fût le premier ou non. Ceci suppose que transformer est l'activité majeure de l'architecte; que pour assumer cette activité projectuelle, il doit connaître l'espace moderne, comprendre sa fluidité, ses lumières, ses transparences, son harmonie, son équilibre, ses surfaces, ses volumétries, ses logiques internes et externes.

Dans ce projet où Fernando Montès dit vouloir «faire une casa Rustici à Paris », une casa Rustici prétendument résumée à sa façade symétrique unifiant par des balcons sublimes deux villas identiques conçues pour deux frères ennemis, apparaît cette volonté de «transformer l'espace du projet comme s'il faisait partie d'une série». Même si d'après Montès, «toutes les grandes villes du monde ont une casa Rustici, toutes sauf une : Paris », ce n'est pas à cette série qu'il semble affilier son projet mais à l'œuvre de Rob Mallet-Stevens (1886-1945) décrit comme «un Terragni moins tragique » et à ses disciples et contemporains, tous engagés en faveur du logement moderne et de la collaboration avec les artistes. Ginsberg (1905-1983) a été son étudiant à l'École spéciale d'architecture (19241929), Chareau (1883-1950) collabore avec lui dans les films de Marcel L'Herbier, L'Inhumaine (1924) et Le Vertige (1927); il participe à la décoration de la villa Noailles (19251928). Il est aussi membre fondateur avec Mallet-Stevens de 1'Union des Artistes Modernes en 1929 qu'intégrera Elkouken (1893-1950) en 1945.
C'est aussi dans les œuvres ultérieures de Fernando Montès que se révèlent les séries qui se croisent dans le projet de l'immeuble de la rue Ramponneau : dans la villa Le Corbusier à Cachaga (Chili) où on retrouve ce totemproue ou, plus tard, à Santiago, où il concevra une place elliptique pour aménager le jardin botanique explorant par-là la série de cette imaginaire place ovale de Vigevano.

Dans son livre La Conception des objets. Son monde de fiction, Daniel Guibert dit s'intéresser à l'activité qui fait être les objets. Pour lui, le projet est un objet spectral.

Ce plan de corrélation [entre intra-système de conception et extra-système de cas passés] agit ainsi, tendu vers un objet spectral, en attente d'existence matérielle, celui du concevoir. Dans ce cas de projection, le spectre se constitue non plus de fragments symboliques de cas passés mais de tout ou partie d'un ou d'édifices idéaux; ils sont fourbis par des doctrines ambiantes, vers lesquelles l'objet spectral sera alors (dys)tendu. Dans ce processus en tendance, l'objet édifice idéal vient symboliquement occuper le lieu d'être de l'objet spectral, composé de multiples graphes orientés, structurés autour de problèmes identifiés.

Heureusement, heuristiquement, l'objet virtuel abandonne la dimension spectrale au fur et à mesure que la générativité créatrice d'émergences métabolise à la fois les matériaux du «faire référence à des cas passés » ou venant d'un édifice idéal qui s'y substitue, ses propriétés convoquées comme finalité empathique ${ }^{28}$.

À cette acception physique du spectre - où émerge la figure du faisceau - vient se greffer, comme par coalescence, une acception barthesienne. On se souvient de Roland Barthes, dans La Chambre claire, cherchant à saisir, presque à surprendre, comme par touches, l'être - tout en dérobement - de la photographie :

L'Operator, c'est le photographe. Le Spectator, c'est nous tous qui compulsons, dans les journaux, les livres, les albums, les archives, des collections de photos. Et celui ou cela qui est photographié, c'est la cible, le référent, sorte de petit simulacre, d'eidôlon émis par l'objet, que j'appellerais volontiers le Spectrum de la Photographie, parce que ce mot garde à travers sa racine un rapport au «spectacle» et y ajoute cette chose un peu terrible qu'il y a dans la photographie : le retour du mort 29 .

28. Daniel Guibert, La Conception des objets. Son monde de fiction, Paris, L'Harmattan, 2002, p. 315.

29. Roland Barthes, La Chambre claire. Note sur la photographie, Paris, Éditions de l'Étoile, Gallimard, 1980, p. 22-23. 
Ainsi, dans le cas de cet immeuble de la rue Ramponneau, l'ante-projet apparaît dans sa dimension spectrale où se conjuguent les figures du faisceau, du spectacle et du retour du mort. Il est la scène d'un spectacle - la genèse du projet éclairée par les mots de Montès où les morts reviennent pour se recomposer, selon des trajectoires voisines avant de se rejoindre, tendus d'une part, par le désir régénérateur de l'architecte, d'autre part, par l'ensemble des contraintes de la commande et de la situation spécifique de cette opération de réhabilitation de l'îlot Ramponneau-Bisson.

À travers cette exogenèse foisonnante dont seul l'anteprojet donne la véritable mesure, semble se profiler la question que Montès formule à propos de Mallet-Stevens : «Sommes-nous en face d'une production ordinaire découlant d'un mode de production d'exception ? 30 » Cet édifice, somme toute lui-même plus « ordinaire » qu' «héroïque», ne serait-il pas une énième preuve de la fascination de Montès pour l'architecte, en même temps qu'un énième mode de référenciation à son œuvre?

30. Fernando Montès, «Case "vitesse” et coin obscur», art. cit., p. 64. 
Olfa Meziou Baccour est diplômée de l'École nationale d'architecture et d'urbanisme (ENAU) de Tunis. Elle enseigne la méthodologie de projet et la critique architecturale. Sa recherche porte sur la genèse de projets architecturaux et la modélisation du processus de conception. Elle a exposé ses travaux dans plusieurs colloques et les a publiés. Membre de l'Association tunisienne de poïétique et d'esthétique (ATPE), elle entretient des liens de collaboration avec l'ITEM dans le cadre d'une recherche plus vaste sur les actes de la création en général (littérature, peinture, architecture...).

Olfa.meziou2014@gmail.com

\section{Exogenèse d'un projet architectural : à la lumière des entretiens avec Fernando Montès}

Cette recherche, qui porte sur la genèse d'un immeuble d'habitation sis au 20-28 de la rue Ramponneau, conçu en 1986 par l'architecte franco-chilien Fernando Montès pour la Régie immobilière de la Ville de Paris, pose la question de la place et du statut de l'entretien en génétique architecturale. L'analyse de cette genèse, entamée in vivo en 1986 et reprise en 2001, a en effet donné lieu à deux séries d'entretiens avec le concepteur. En revenant sur son projet, quinze ans plus tard, l'architecte apporte de nouveaux contenus qui permettent d'éclairer différemment le travail de conception, et notamment d'ouvrir de nouvelles pistes exogénétiques en matière d'identification des sources. Moyennant une nouvelle enquête, ces données permettent de renouveler l'interprétation des documents génétiques en révélant, d'une part, des modes inédits de constitution du matériel référentiel, et d'autre part, en explicitant le fonctionnement de ces procédures référentielles conçues comme une façon de donner forme à la source (à ce qui est prélevé dans l'emprunt) et comme sa transformation motivée par un faisceau de désirs et de contraintes.

Focused on the construction of a residential building located at 20-28 rue Ramponneau, designed in 1986 by the Franco-Chilean architect Fernando Montès for the Régie immobilière de la Ville de Paris, this article investigates the place and status of interviews in architectural genetics. The analysis of this construction, started in vivo in 1986 and resumed in 2001, has in fact given rise to two series of interviews with the designer. Looking back on his project fifteen years after it had begun, the architect shed new light on the design work and opened up new exogenetic routes for identifying sources. These findings make a new investigation possible. The reinterpretation of genetic documents reveals new methods of gathering the referential material, as well as the explication of the operation of referential procedures designed both to give shape to the source (to the borrowed material) and to transform it through a network of desires and constraints.

Dieser Beitrag, der sich mit der Entstehung eines Wohngebäudes in der rue Ramponneau 20-28 auseinandersetzt, das 1986 von dem französisch-chilenischen Architekten Fernando Montès für die Régie immobilière de la Ville de Paris entworfen wurde, wirft die Frage nach dem Ort und dem Status der Instandhaltung in der Architekturgenetik auf. Die Analyse dieser Genese, die 1986 in vivo begonnen und 2001 wieder aufgenommen wurde, hat in der Tat Anlass zu zwei Interviewreihen mit dem Designer gegeben. Im Rückblick auf sein Projekt, fünfzehn Jahre später, bringt der Architekt neue Inhalte ein, die ein neues Licht auf die Entwurfsarbeit werfen und insbesondere neue exogenetische Wege für die Identifizierung von Quellen eröffnen. Diese Daten ermöglichen es, die Interpretation der genetischen Dokumente zu erneuern, indem sie einerseits neue Wege der Konstituierung von Referenzmaterial aufzeigen und andererseits die Funktionsweise dieser Referenzverfahren erklären, die als eine Art der Formgebung der Quelle (dessen, was der Referenz entnommen wird) und als ihre durch ein Bündel von Wünschen und Zwängen motivierte Umformung gedacht sind.
Esta investigación, dedicada a la génesis de un edificio residencial ubicado en la calle Ramponneau nos 20-28, concebido en 1986 por el arquitecto franco-chileno Fernando Montès para la Red Inmobiliaria de la Ciudad de París, plantea la cuestión de la ubicación y el papel de la entrevista en genética arquitectural. El análisis de esta génesis, comenzada in vivo en 1986 y retomada en 2001, ha dado lugar a dos series de entrevistas con el creador. Reflexionando de nuevo sobre su proyecto, quince años más tarde, el arquitecto aporta nuevos contenidos que permiten iluminar con otra perspectiva el trabajo de concepción y, en particular, abrir nuevas pistas exogenéticas en lo que respecta la identificación de las fuentes. A través de una nueva investigación, estos datos permiten enriquecer la interpretación de los documentos genéticos, develando, por una pate, modos inéditos de constitución del material referencial y, por la otra, explicitando el funcionamiento de esos procedimientos referenciales concebidos como una manera de dar forma a la fuente (al elemento que se toma prestado) y como su transformación motivada por un haz de deseos y de restricciones.

Esta investigação, que se debruça sobre a génese de um edifício residencial situado na rue Ramponneau 20-28, concebido em 1986 pelo arquiteto franco-chileno Fernando Montès para a Régie immobilière de la Ville de Paris, levanta a questão do lugar e do estado de manutenção em genética arquitetónica. A análise desta génese, iniciada in vivo em 1986 e retomada em 2001, deu, de facto, lugar a duas séries de entrevistas com o autor. Regressando ao seu projeto, quinze anos mais tarde, o arquiteto traz novos conteúdos que permitem lançar uma nova luz sobre o trabalho de conceção e, designadamente, abrir novos caminhos exogenéticos em termos de identificação de fontes. Através de uma nova investigação, estes dados permitem renovar a interpretação dos documentos genéticos, revelando, por um lado, formas inéditas de constituição do material de referência, e, por outro lado, explicitando o funcionamento desses procedimentos de referência concebidos não só como um modo de dar forma à fonte (ao que foi aproveitado no empréstimo), mas também como a sua transformação motivada por um conjunto de desejos e constrangimentos.

Questa ricerca, incentrata sulla genesi di un immobile abitativo sito al numero 20-28 di rue Ramponneau, concepito nel 1986 dall' architetto franco-cileno Fernando Montès per la Régie immobilière de la Ville de Paris, pone la questione del ruolo e dello statuto dell'intervista nella genetica architetturale. L'analisi di questa genesi, cominciata in vivo nel 1986 e ripresa nel 2001, ha dato luogo, in effetti, a due serie d'interviste con l'ideatore. Tornando sul suo progetto, quindici anni più tardi, l'architetto apporta nuovi contenuti che permettono di gettare una nuova luce sul lavoro di concezione, e in particolar modo di aprire nuove piste esogenetiche per l'identificazione delle fonti. Attraverso una nuova inchiesta, questi dati permettono di rinnovare l'interpretazione dei documenti genetici rivelando, da un lato, modi inediti di costituzione del materiale di riferimento ed esplicitando, dall'altro, il funzionamento di queste procedure di riferimento concepite come un modo di dar forma alla fonte (a ciò che viene prelevato nel prestito) e come la sua trasformazione motivata da un insieme di desideri e di vincoli. 